\title{
In Vitro Dosimetry of Agglomerates
}

\author{
V. Hirsch,${ }^{a \S}$ C. Kinnear, ${ }^{a \S}$ L. Rodriguez-Lorenzo, ${ }^{a \S}$ C. A. Monnier, ${ }^{a}$ B. Rothen- \\ Ruthishauser, ${ }^{a}$ S. Balog ${ }^{a}$ and A. Petri-Fink ${ }^{a, b^{*}}$ \\ Agglomeration of nanoparticles in biological fluids is a pervasive phenomenon that leads to \\ difficulty in the interpretation of results from in vitro exposures, primarily due to the \\ differing particokinetics of agglomerates to nanoparticles. Therefore, well-defined small \\ agglomerates were designed that possessed different particokinetics profiles, and their \\ cellular uptake was compared to a computational model of dosimetry. The approach used \\ here paves the way for a better understanding of the impact of agglomeration on the \\ nanoparticle-cell interaction.
}

\section{Introduction}

Engineered nanoparticles meet the biological world at the nanobio interface. This encounter holds many promises, such as applications in medicine or the sensing of biomarkers, and has led to novel science investigating the cellular interaction of nanoparticles (NPs). ${ }^{1}$ This interaction is routinely assessed in vitro due to the need for alternatives to invasive animal experimentation, specifically relating to influential concept first proposed in 1959: reduction, refinement, and replacement of animal use. ${ }^{2}$

In the last decade a great deal of effort has been dedicated to elucidate the impact of physicochemical properties of NPs, such as their size, surface charge, hydrophobicity, or shape, on their subsequent cellular interaction. In line with in vitro testing of soluble compounds, the dose that cells are exposed to is frequently assumed to equal the concentration of NPs in suspension. ${ }^{3,4}$ However, compared to soluble compounds, NPs exhibit a wide range of diffusion coefficients and sedimentation velocities depending on both their size and density, which can result in vastly differing applied doses. ${ }^{5}$ Teeguarden et al. considered the sedimentation and diffusion of NPs as the major factors that determine the interaction with the cellular membrane in cell culture media. ${ }^{6}$ Together with Hinderliter, they have developed a model (i.e. the "In Vitro Sedimentation, Diffusion and Dosimetry model", ISDD) to study the movement of NPs in cell culture media and their respective particokinetics (i.e. rate of delivery of NPs to cells in vitro). ${ }^{7}$ Complementary to this, Cho et al. showed by administrating gold nanoparticles to an inverted in vitro cellular system that, above a certain ratio of diffusion to sedimentation velocities, sedimentation was the cause of an anomalously higher cellular uptake. $^{8}$

These studies, among others, have outlined an approach for expressing the applied dose, in vitro, of colloidally stable single NPs. However, before NPs can interact with living cells/organisms, their surfaces are exposed to biological fluids such as cell culture medium, blood, or lung fluid, whose components interact with the particle surfaces. ${ }^{9}$ This has two major consequences: First, it results in a tightly bound immobile protein layer formed on the particle surface (the socalled hard corona) and possibly a weakly associated mobile layer (the soft corona). ${ }^{10,11}$ Second, it may induce NP agglomeration, which is a common phenomenon in this complex environment and consequently has to be taken into account. $^{12}$ In spite of this, we are currently incapable of accurately predicting the colloidal behaviour of NPs in cell culture medium, which contains a large variety of proteins, small molecules, and ionic salts, all of which can affect the hydrodynamic behaviour of NPs. ${ }^{13-16}$ Agglomeration in cell culture media leads to misrepresentative results and impedes experimental reproducibility, ${ }^{17,18}$ not least due to the fact that often the agglomerates are ill-defined and irregularly shaped. ${ }^{19}$ The necessity of studying agglomerates in vitro is highlighted by various studies, where single and agglomerated NPs were shown to exhibit different cellular uptake and cytotoxicity profiles. ${ }^{17,18,20}$ In order to validate the approach of particokinetics, salt is most often used to induce agglomeration that leads to near-micron sized agglomerates or, more recently, the application of serum proteins can be used to halt the agglomeration at defined times. ${ }^{21-23}$ These large, often anisotropic and polydisperse, agglomerates are normally characterized by standard dynamic light scattering (DLS), 
however rotational diffusion can result in additional decays in the autocorrelation function which is angle dependent, complicating the interpretation of DLS from agglomerates. ${ }^{24,25}$ Another difficulty arises from the measuring of the fractal dimension and packing factors of agglomerates, whereby all previous publications regarding particokinetics needed to make assumptions regarding these factors. ${ }^{7,21,22}$

In this report, we aim to isolate and characterize factors that define the transport profile of agglomerates in vitro through the study of well-controlled and defined small agglomerates, thereby giving a more general approach to study analogous systems. To this end, we designed and synthesized small agglomerates of gold NPs using a method of $\mathrm{pH}$-induced and polymer terminated "start-stop" self-assembly. Through altering the kinetics of agglomeration and the "stop" point, we were able to tune both the size and density of the agglomerates. Thorough characterization, for the first time, by depolarized dynamic light scattering (DDLS) and cryo-TEM enabled the precise measurement of key parameters that define their transport profile in vitro, without needing to make assumption regarding the fractal dimension or packing fraction. ${ }^{25}$ This system of small agglomerates mimics the transport profile of more common "collapsed" colloidal systems and further validates the application of particokinetics to agglomerates in vitro. As a consequence of this well-characterized system, the quantitative description of the impact of agglomeration on the rate of cellular association in a model cancer cell line could additionally be elucidated.

\section{Experimental Section}

All Chemicals were purchased from Sigma Aldrich, Switzerland and of analytical reagent grade, used without additional purification. Water refers in all synthesis steps to ultrapure deionized water of $18 \mathrm{M} \Omega \cdot \mathrm{cm}$ (Millipore $\mathrm{AG}$, Switzerland).

\section{Synthesis of Tiopronin coated Gold Nanoparticles}

Citrate capped Au-NPs were prepared according to the protocol of Turkevich et al. ${ }^{26}$ Briefly, a $0.5 \mathrm{mM}$ aqueous tetrachloroauric acid $\left(\mathrm{HAuCl}_{4} \times 3 \mathrm{H}_{2} \mathrm{O}\right)$ solution was boiled for $20 \mathrm{~min}$ in the presence of $1.5 \mathrm{mM}$ sodium citrate $\left(\mathrm{C}_{6} \mathrm{H}_{5} \mathrm{O}_{7} \mathrm{Na}_{3}\right)$. Tiopronin $\left(\mathrm{C}_{5} \mathrm{H}_{9} \mathrm{NO}_{3} \mathrm{~S}\right)$ was then added at a concentration of $0.015 \mathrm{mM}$ to the solution at room temperature. The Au-NPs had an average diameter of $14.3 \pm 1.2 \mathrm{~nm}$, as measured by TEM.

\section{pH Controlled Agglomeration of Tiopronin Au-NPs}

The agglomeration of the tiopronin coated Au-NPs (Tio-AuNPs) was induced by adding increasing amounts of $1 \mathrm{M} \mathrm{HCl}$ solution to each $30 \mathrm{ml}$ Tio-Au-NP suspension. The $\mathrm{pH}$ was monitored (VWR ${ }^{\circledR}$ sympHony ${ }^{\mathrm{TM}}$ meter) before stabilizing the single NPs and agglomerates by addition of $3 \mathrm{~mL}$ of a mixture of a $10 \% \mathrm{w} / \mathrm{v}$ aqueous solution of polyvinyl alcohol (Mowiol 383 , Omya AG, Switzerland) and a $2 \% \mathrm{w} / \mathrm{v}$ aqueous solution of vinylalcohol/vinylamine copolymer (Erkol S.A, Spain) in a ratio of 9:1. The polymer solutions were prepared by dissolving the powder in water, followed by rapidly heating the solution to $90^{\circ} \mathrm{C}$ for $15 \mathrm{~min}$ and cooling to RT. After four days of storage at $4{ }^{\circ} \mathrm{C}$, the different $\mathrm{NP}$ suspensions were washed by centrifugation. The suspensions were centrifuged at 10,000 $\times \mathrm{g}$ (single NPs) and 5,000 $\times \mathrm{g}$ (agglomerates) for $1 \mathrm{~h}$, the supernatant was collected and centrifuged again under the same conditions. This process was repeated three times, with the NP and agglomerates collected and re-dispersed in a total of $15 \mathrm{~mL}$ each.

\section{Characterization}

Isothermal Titration Microcalometry (ITC). Characterization of the tiopronin interaction with the Au-NP surface was probed by ITC (MicroCal VP-ITC). Measurements were carried out in water at $25^{\circ} \mathrm{C}$ by titrating a $0.5 \mathrm{mM}$ solution of tiopronin into a $14.7 \mathrm{nM}$ suspension of Au-NPs (equal to $1 \mathrm{mM}$ of gold). After baseline equilibration, a first injection of $3 \mu \mathrm{L}$ was followed by injections of $4 \mu \mathrm{L}$ at intervals of $1800 \mathrm{sec}$, with constant stirring of the Au-NP suspension at $242 \mathrm{rpm}$. The reference power was set to $10 \mu \mathrm{cal} / \mathrm{sec}$.

UV-Vis Spectroscopy. UV-Vis spectra of the single PVA coated Tio-Au-NPs (single Au-NPs) and the prepared agglomerates (agglomerates-1 or agglomerates-2) were recorded using a Jasco V670 spectrophotometer. The samples were diluted 1 fold in water, PBS or cell culture medium.

The self-assembly kinetics were studied by UV-Vis and DLS. Samples were prepared by adding $1 \mathrm{M} \mathrm{HCl}$ solution to $2 \mathrm{~mL}$ of Tio-Au-NPs suspension with a gold concentration of $0.5 \mathrm{mM}$. The suspension was homogenized by inversion and the selfassembly followed immediately by UV-Vis and DLS.

Sample vitrification and Cryo-Transmission Electron Microscopy (Cryo-TEM). $5 \mu 1$ of sample solution were first deposited on a carbon-coated copper grid (Lacey carbon film grids, Plano $\mathrm{GmbH}$ ). Following this, excess liquid was carefully blotted away with filter paper (Whatman qualitative filter paper, grade 1) before plunging the sample into a liquid ethane bath cooled by liquid nitrogen. The vitrified specimens were then kept for storage in liquid nitrogen and analyzed the following day.

All grids were investigated with a FEI Tecnai F20 cryotransmission electron microscope with an operating tension of $200 \mathrm{kV}$. Images were recorded under low-dose conditions with an UltraScanTM 1000 CCD sensor (Gatan, Inc.) with an image resolution of $2048 \times 2048$ pixels.

Depolarized Dynamic Light Scattering (DDLS). Coherent laser light becomes partially depolarized upon scattering from gold NPs, and depolarization provides a tool to probe rotational diffusion. Therefore, we used DDLS to estimate the hydrodynamic diameters of the Au-NPs and their agglomerates in water at a concentration of $100 \mu \mathrm{g} / \mathrm{mL}$. Temporal correlation functions were recorded at three angles: $\theta=60^{\circ}, 90^{\circ}$ and $120^{\circ}$, using a goniometer setup (LS Instruments, Switzerland) equipped with a He-Ne laser $(\lambda=632.8 \mathrm{~nm})$. The depolarized mode was detected via a cross-polarizer.

\section{Cell culture and Exposure}

Human cervix carcinoma cells (HeLa cells) were purchased from HPA Culture Collections UK and cultured in $75 \mathrm{~cm}^{2}$ cell culture flask (Techno Plastic Products AG, Switzerland) using 1x Dulbecco's Modified Eagle Medium (DMEM, Invitrogen, Switzerland) supplemented with $10 \%$ Fetal Bovine Serum (FBS) and $1 \%$ Penicillin/Streptomycin (Invitrogen, Switzerland) at $37{ }^{\circ} \mathrm{C}$ and $5 \% \mathrm{CO}_{2} .24 \mathrm{~h}$ prior to exposure cells were detached using Trypsin-EDTA (Invitorgen, Switzerland), seeded at a density of $2.5 \times 10^{4}$ cells per well in a 48 well-plate 
(Costar, Corning Incorporated, USA) and cultured at $37^{\circ} \mathrm{C}$ and $5 \% \mathrm{CO}_{2}$

On day of exposure cells were washed with 1x PBS, single AuNPs and according agglomerates were added at concentrations of $100 \mu \mathrm{g} / \mathrm{mL}$ of Au diluted in $10 \%$ FBS supplemented cell culture medium and incubated for $0.5,1,6,12,24$ and $32 \mathrm{~h}$ at $37^{\circ} \mathrm{C}$ and $5 \% \mathrm{CO}_{2}$. For subsequent evaluation of the cell viability, supplemented cell culture medium acted as the negative control and the detergent Triton X-100 at a concentration of $0.2 \%$ in PBS acted as the positive control. Each exposure was repeated a total of 5 times $(n=5)$.

\section{Cell Uptake}

It should be noted that we use the term "uptake" hereafter instead of the term "cell associated material" for brevity, however through previous experience, we have observed that washing with PBS is sufficient to remove NPs loosely adsorbed to the cell membrane. Cell uptake of single Au-NPs and agglomerates was measured by Inductively Coupled Plasma Optical Emission Spectroscopy (ICPOES, PerkinElmer Optima 7000 DV). Briefly, after exposure the cells were washed three times with $1 \mathrm{x}$ PBS and the cells were dissolved in aqua regia $\left(\mathrm{HNO}_{3}: \mathrm{HCl}\right.$ with volume ratio of 1:3, 500 $\mu \mathrm{L} /$ well), transferred to $15 \mathrm{~mL}$ Falcon tubes (BD Biosciences, Switzerland) and diluted to $10 \mathrm{~mL}$ with water. The treated samples were then measured in quintuplicate by ICP-OES. A standard curve of aqueous $\mathrm{Au}$ solutions was recorded to quantify the amount of intracellular Au. All solutions were measured in triplicate.

\section{Cytotoxicity}

A Cytotoxicity Detection Kit (Roche Applied Science, Germany) was used to quantify the level of cytotoxicity following the exposure to single Au-NPs and agglomerates. The calorimetric assay is based on the measurement of the lactate dehydrogenase (LDH) activity, an enzyme released into the cell culture medium as an indicator of damaged cell membranes. ${ }^{27}$ Supernatants were measured in triplicate at $490 \mathrm{~nm}$ (with a reference of $630 \mathrm{~nm}$ ) using a multi-plate spectrometer (Benchmark Plus, Bio-Rad, Switzerland).

\section{Results and Discussion}

More specifically, citrate-capped gold nanoparticles (Au-NPs) were covalently functionalized with $\mathrm{N}-(2-$ mercaptoproionyl)glycine (tiopronin). ${ }^{28}$ The surface of the NPs was coated with an amount of tiopronin that corresponds to one near-monolayer as determined by isothermal titration calorimetry (ITC; Fig. S1). Therefore, there was no excess tiopronin in solution allowing a precise control over the subsequent Au-NP assembly. The terminal carboxylic acid then allowed controlled agglomeration through hydrogen bonding. The agglomeration was promoted by decreasing the $\mathrm{pH}$, leading to increased protonation of the carboxylic group and decreased electrostatic stabilization in favour of hydrogen bonds between the tiopronin molecules (Fig. 1). ${ }^{29}$ The controlled agglomeration was monitored by UV-Vis spectroscopy where a broadening and red-shift of the localized surface plasmon resonance (LSPR) of single Au-NPs indicates agglomeration (Fig. S2). ${ }^{30}$ Complementary to this, the influence of $\mathrm{pH}$ on kinetics of self-assembly was followed with DLS, and limiting regimes were found for either diffusion or reaction limited cluster aggregation at lower or higher $\mathrm{pH}$ respectively (Fig S3). These regimes are well-known to result in different densities of agglomerates, therefore allowing the design of similar sized agglomerates with differing densities. ${ }^{31,32}$

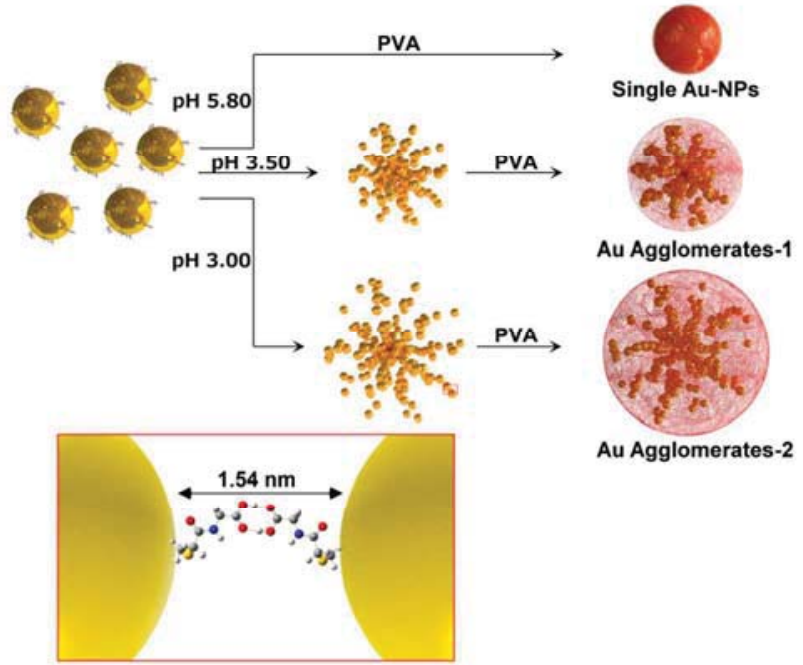

Fig.1. Preparation of PVA-coated, tiopronin-modified Au-NPs: Decreasing the $\mathrm{pH}$ induces the agglomeration of tiopronin-modified Au-NPs while adding PVA results in the formation of a stabilizing polymer shell. Inset: illustration of tiopronin-induced agglomeration model triggered by hydrogen bonding interactions. The lowest energy conformation of tiopronin dimer, calculated using DFT method (Gaussian 03, no nearest-neighbor interactions included), provides a surface-to-surface gap of $1.54 \mathrm{~nm}$ allowing a strong interaction between NPs.

This agglomeration was then completely quenched by simple addition of a mixture of polyvinyl alcohol and polyvinyl alcohol-vinylamine co-polymer (hereafter referred to as PVA) whose amine functionality interacts with the negatively charged tiopronin coated Au-NPs. ${ }^{33}$ Thus, agglomeration can be halted at any stage, allowing control over the mass density and size of the agglomerates. In addition, the PVA coating stabilized the agglomerates in physiological conditions and was necessary to avoid the reversal of the self-assembly upon increase of the $\mathrm{pH}$ to physiological levels. ${ }^{34}$ Crucially, the biocompatibility of both tiopronin and PVA is well established therefore tiopronin and PVA not only play critical roles in controlling the agglomeration of the Au-NPs but also provide a biocompatible shell. ${ }^{35-38}$ Notably, all systems displayed the same surface properties, therefore we assume that the PVA coating will have a similar influence on both single NPs and agglomerates in their interactions with the cell membrane.

The suspension of functionalized Au-NPs was stable at $\mathrm{pH}$ values higher than 3.8 whereas the LSPR band broadened and red-shifted below pH 3.5 with the rate of change of the LSPR band increasing with decreasing $\mathrm{pH}$ to a lower limit of $\mathrm{pH} 2.5$ (Fig. S2). This difference in the rate of agglomeration allowed the controlled synthesis of agglomerates with different sizes and densities. Moreover, the single NPs and agglomerates were stable in physiological conditions, as assessed by UV-Visible spectroscopy where similar LSPR bands were observed in water and cell culture media (DMEM) (Fig. 2 and S4). In agreement with the UV-Vis results, cryo-TEM images show mostly well dispersed single NPs at $\mathrm{pH} 5.8$, while an increased degree of agglomeration was observed at lower $\mathrm{pH}$ values (Fig.2B-C). The hydrodynamic diameters of the single NPs and agglomerates were measured by Depolarized Dynamic Light 
Scattering (DDLS) (Table 1), where the intensity weighted polydispersity was described by a Schulz-Zimm distribution (Fig. S5).
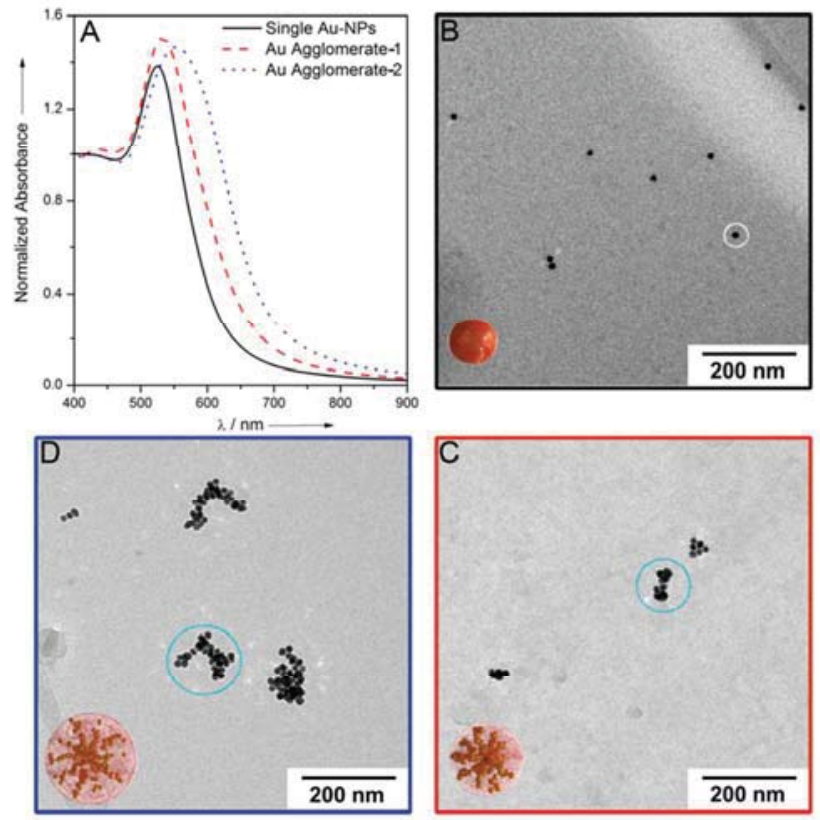

Fig.2. (A) Extinction spectra of the three systems studied after dilution in DMEM. The spectra were normalized based on their absorbance at $400 \mathrm{~nm}$. (B-D) Cryo-TEM images of (B) single Au-NPs, (C) Au agglomerates-1, and (D) Au agglomerates-2, where the solid circle represents the hydrodynamic diameter measured by DDLS.

Table 1. Hydrodynamic diameter of the investigated systems.

\begin{tabular}{lcc}
\hline \multicolumn{1}{c}{ Suspensions } & $\mathbf{d}[\mathbf{n m}]$ & $\begin{array}{c}\text { Polydispersity } \\
{[\%]}\end{array}$ \\
\hline Tiopronin-Au-NPs & 19 & 20 \\
Single Au-NPs & 65 & 22 \\
Agglomerates-1 & 145 & 57 \\
Agglomerates-2 & 168 & 60 \\
\hline
\end{tabular}

The NP-cell interaction of single Au-NPs and their agglomerates was tested on a HeLa cell line exposed at a mass concentration of $100 \mu \mathrm{g} / \mathrm{mL}$. Cellular toxicity, as assessed by the released lactate dehydrogenase (LDH) after $24 \mathrm{~h}$ exposure, did not change with increased agglomeration (Fig. S7). In agreement with our result, Albanese and Chan also reported that the viability of HeLa cells was not affected by the presence of transferrin coated $\mathrm{Au}$ agglomerates. ${ }^{17}$ The intracellular $\mathrm{Au}$ content as a function of time was measured by Inductively
Coupled Plasma Optical Emission Spectroscopy (Fig. 3). If this data were to be interpreted without consideration of particokinetics, then the conclusion would be drawn that single NPs are taken up faster than agglomerates-1 which themselves result in slower uptake kinetics than agglomerates-2.

However, due to the fact that colloids will be transported to the cell surface at different rates depending on their size and density, a further step of interpretation is needed.

Cellular uptake is a complex process, which involves three elementary stages: (i) transport of NPs to the cell, (ii) adsorption of NPs to the cell membrane, and (iii) internalization of the NPs. For the studied single NPs and agglomerates, all having the same surface properties (e.g. polymer coating and surface charge), the uptake pathways will not change because of the surface chemistry (in general, receptor-mediated endocytosis). ${ }^{39}$ In addition, it has been shown that the upper limit of endocytosis for HeLa cells is approximately $250 \mathrm{~nm}$ in diameter, meaning both single NPs and agglomerates would be internalized in our system. ${ }^{16}$ Therefore, it is possible that the transport properties trigger the differences in uptake kinetics. Evidence for this comes from the reports of Teeguarden et al. and Cho et al. who both showed the importance of sedimentation and diffusion, however, the role of agglomeration has been largely overlooked. ${ }^{8,40}$ Agglomerates are not solid particles due to fractal structure (space between individual packed NPs), and therefore they have a lower mass density than NPs with comparable size, affecting on their motion profile. ${ }^{41}$ Zhu et al. calculated that single NPs of $80 \mathrm{~nm}$ settle between 9 to 17 times faster than agglomerates of the same size, which clearly highlights the vastly different cases of agglomerates and solid NPs. ${ }^{42}$ We determined the diffusion coefficients and sedimentation velocities through the average hydrodynamic diameter and effective mass density of our systems (Table 1, S2, and S3). It was found that the sedimentation velocity increased in the following order: single NPs $<$ agglomerates- $1<$ agglomerates-2. However, with this data we would expect different uptake kinetics with respect to that observed. This disparity is due to the combination of sedimentation, which is assumed to be time-independent i.e. terminal velocity is reached instantaneously, and diffusion which is a time dependent process. Therefore, a model needs to be used to evaluate the relative importance of sedimentation compared to diffusion in closed systems such as in vitro cell exposures.

Computational modelling can be used to estimate the delivered dose as a function of time and transport properties (diffusion and sedimentation) in order to understand the consequence of agglomeration in cell uptake. This application has been highlighted by the work of Hinderliter et al. ${ }^{7}$ where a model was presented (ISDD model) that estimates the particokinetics and dosimetry of non-interacting NPs in cell culture media as a 

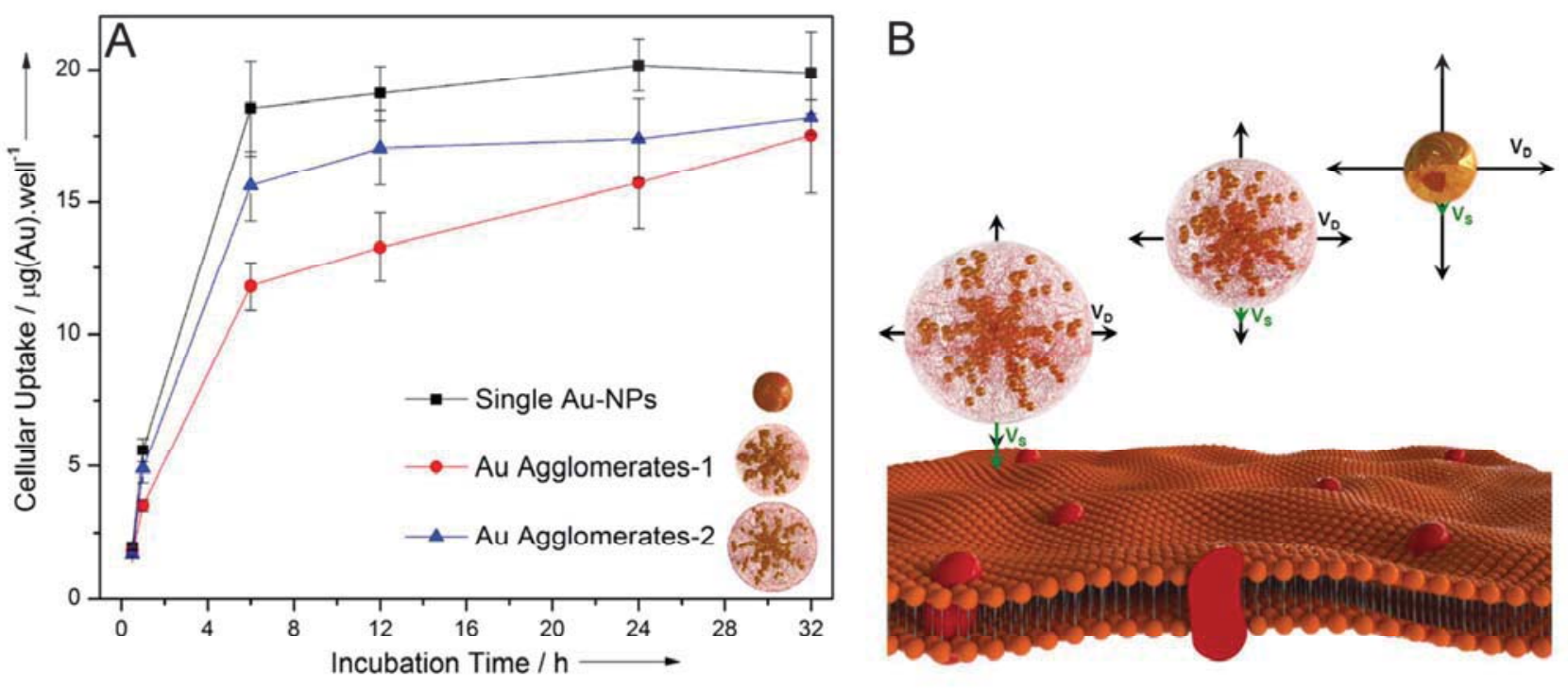

Fig.3. (A) Quantification of intracellular Au content by ICP-OES shows the influence of cellular uptake as a function of the incubation time of single Au-NPs (black), agglomerates-1 (red), and agglomerates-2 (blue). HeLa cells were incubated with $100 \mu \mathrm{g} / \mathrm{mL}$ NPs or agglomerates, respectively $(\mathrm{n}=5$, Error Bars: SD). (B) Illustration of sedimentation velocity of single NPs and their agglomerates.

function of time. To estimate the time-dependent cellular dose from NPs, Hinderliter et al. adapted the equation of motion of monodisperse NPs, governed by sedimentation and diffusion, postulating that from the point of view of cellular dose only the vertical movement is relevant while the lateral movement of the particles is not:

$$
\frac{\partial \mathrm{n}(\mathrm{x}, \mathrm{t})}{\partial \mathrm{t}}=\mathrm{D} \frac{\partial^{2} \mathrm{n}(\mathrm{x}, \mathrm{t})}{\partial \mathrm{x}^{2}}-\mathrm{V} \frac{\partial \mathrm{n}(\mathrm{x}, \mathrm{t})}{\partial \mathrm{x}}
$$

where $\mathrm{D}$ and $\mathrm{V}$ are the diffusion coefficient and sedimentation velocity of the NPs respectively, and $\mathrm{n}$ is the NP concentration. In order to solve the equation of motion, the following initial and boundary conditions were used: at the beginning of the experiment the NPs are homogenously distributed in the fluid, and as soon as a NP contacts the cell surface it becomes internalized instantly by the cell and remains within the cell. With these conditions, the partial differential equation (PDE), Equation (1), was solved using the partial differential equation solver in Matlab ${ }^{\circledR}$ (Mathworks, Inc.), giving the number of particles present at the bottom of the well as a function of time. Despite its merits, the ISDD model per se is idealistic compared to real in vitro experiments since the initial and boundary conditions are simplified (more details in the supporting information). In particular, the rate of NP internalization is a function of size and surface chemistry, and internalization is not instantaneous. An optional "sticky" boundary condition has been coded within the ISDD model relating to a probability of the NP adsorbing or not to the modelled cell layer. However, there is little quantitative data regarding the magnitude of this factor in the literature, and it would be logical to assume that it depends strongly on the surface chemistry of the NPs. As an attempt to minimize the deviation stemming from variable exposure conditions, a special emphasis was given to ensuring identical experimental procedures across exposures. Therefore, if the relative ratios of cellular dose between different NPs and agglomerates are considered, the deviations resulting from the non-ideal exposure conditions are minimized allowing a more accurate prediction of cellular doses with the ISDD model.

The ISDD model is able to reproduce the general trends in the relative ratio of uptake for the three systems, as shown in Fig. 4. The non-ratiometric experimental data and model are given in the Supplementary Information. In the first two cases (Fig. 4A and 4B), the single NPs are taken up quicker and to a greater extent than either of the agglomerates although the difference is most evident for the agglomerates-1, which have a lower density and therefore a lower sedimentation velocity than agglomerates-2. A common feature of Figure 4B and Figure 4C is the first data point that represents the initial uptake, which is similar, i.e. close to 1 , for all systems. This similarity of uptake is well explained by the modelled transport processes, which further substantiates the hypothesis that transport processes dictate the uptake of chemically-similar NPs.

Despite the consistency between the model and the experimental ratios, the differences in measured uptake between agglomerates could partly originate from different uptake kinetics as discussed by Sharma et al. This is typically not true for very large differences, as shown by Dos Santos et $a l$. where HeLa cells, among others, were found to exhibit dif uptake kinetics dependent on NP sizes, although there was no consideration given to the variations in diffusion coefficients with particle size. ${ }^{43}$ However, a number 

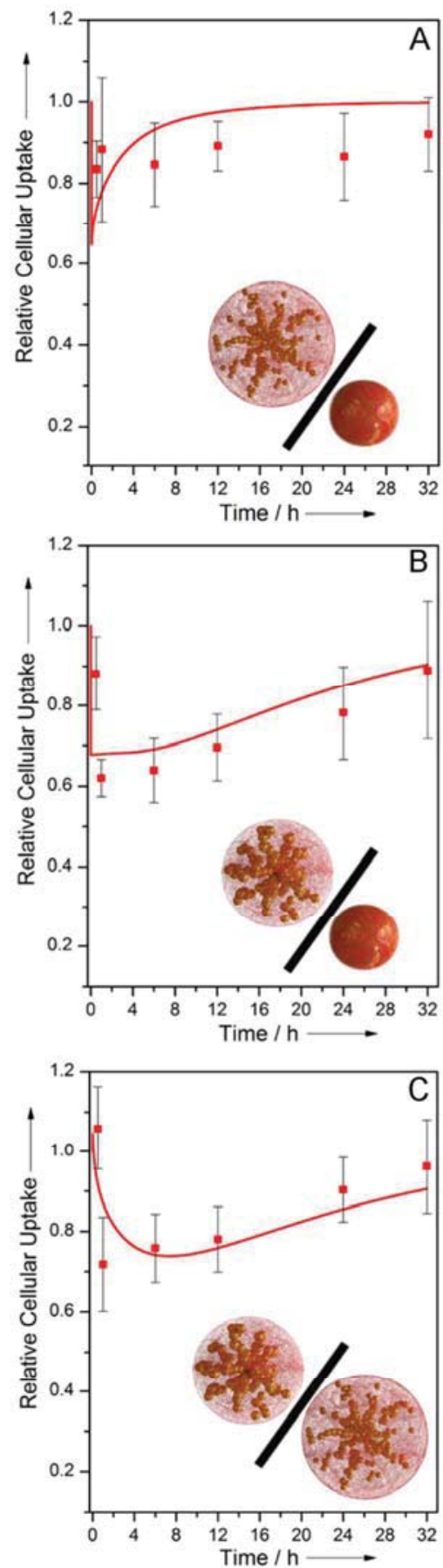

Fig.4. Ratio of NP internalization as function of time as measured by ICP-OES ( $\mathrm{n}=5$; Error Bar $=\mathrm{SD})$. Solid line: ISDD model. (A) $\mathrm{Au}$ agglomerates-2 to single $\mathrm{Au}-\mathrm{NPs}$, (B) Au agglomerates-1 to single AuNPs and (C) Au agglomerates-1 to Au agglomerates-2.

of studies have shown that the key parameter is, in fact, the radius of curvature of the particle upon contact with the cell given that there is no way for the cell to "sense" what the shape of the particle is beyond the point of contact. ${ }^{44-47}$ This highlights an advantage of using agglomerates as opposed to different sized single NPs, namely the radius of curvature for the agglomerates upon contact with the cell is close to that of the individual NPs. To evaluate this hypothesis, a repeated exposure was undertaken using large single gold NPs of identical surface chemistry, with a core diameter of $130 \mathrm{~nm}$ and a hydrodynamic diameter of $200 \mathrm{~nm}$, which exhibited a very high sedimentation velocity. Due to the much lower surface curvature of the large NP, a reduced uptake was measured, despite the fast sedimentation, relative to the agglomerates-1 composed of single NPs (Fig. S8). With consideration of the above points, if we assume that single NPs are taken up faster than the agglomerates, then a slightly higher value would be expected than the model predicts for the ratio of agglomerates to single NPs. This is exactly what is observed in Fig. 4A and Fig. 4B. Considering the best agreement of the model was found for the two agglomerates of similar size but differing density, the conclusion can be drawn that this approach accurately predicts the particokinetics in vitro and provides a more accurate method to test, among other things, size dependent uptake - something which is rarely attempted.

\section{Conclusions}

We have designed well-controlled agglomerates of NPs in order to test the approach of quantifying cellular dose in vitro with a computational model, considering only diffusion and sedimentation. It was found that the model could accurately describe the observed experimental trends in cellular uptake of the NPs or agglomerates, thereby validating this specific approach in the study of NP-cellular interactions. These findings suggest that particokinetics should be taken into account for all in vitro exposures, where interpretation of the relative end-points, such as uptake or cytotoxicity, provides a more robust method to compare NPs rather than the absolute end-point. Nevertheless, more work is needed to understand the impact of inter-particle interactions, especially with highly concentrated systems.

\section{Acknowledgements}

The authors thank Dr. Miguel Spuch-Calvar for drawing Figure 1 and Figure 3b. The support of the Dr. Alfred Bretscher Fund is gratefully acknowledged and access to Cryo-TEM was kindly provided by the Microscopy Imaging Centre of the University of Bern. This work was supported by the Swiss National Science Foundation (PP00P2-123373/1), the Adolphe Merkle Foundation and the University of Fribourg. We are grateful to Dr. Justin G. Teeguarden (Biological Science Division, Pacific Northwest National Laboratory, Richland, WA, USA) for providing the ISDD code.

\section{Notes and references}

${ }^{a}$ Adolphe Merkle Institute, University of Fribourg, Route de 1'Ancienne Papeterie, P.O. Box 209, Marly 1723 (Switzerland).

${ }^{b}$ Department of Chemistry, University of Fribourg, Chemin du Musse 9, Fribourg 1700 (Switzerland).

* Author for correspondence (alke.fink@unifr).

$\S$ These authors contributed equally to this work.

$\dagger$ Electronic Supplementary Information (ESI) available: ITC data for tiopronin/Au-NPs interactions, agglomeration kinetics at different $\mathrm{pH}$ for tiopronin-coated Au-NPs, UV-Vis spectra in water, PBS and DMEM and temporal correlation functions for single Au-NPs and corresponding agglomerates, calculation of diffusion and sedimentation parameters, modelling of relative cell uptake based at ISDD model and cytotoxicity of 
single Au-NPs and their agglomerates, and synthesis and cell uptake of large spherical Au-NPs. See DOI: 10.1039/b000000x/

1 A. Verma and F. Stellacci, Small, 2010, 6, 12-21.

2 W. M. S. Russell and R. L. Burch, The principles of humane experimental technique, UFAW, Potters Bar, 1992.

3 L. Braydich-Stolle, Toxicol. Sci. 2005, 88, 412-419.

4 J. G. Hardman, L. S. Goodman and A. G. Gilman, Goodman \& Gilman's The pharmacological basis of therapeutics, McGraw-Hill Med. Publ, New York, NY, 10th edn., 2001.

5 W. J. Stark, Angew. Chem. Int. Ed., 2011, 50, 1242-1258.

6 J. G. Teeguarden, P. M. Hinderliter, G. Orr, B. D. Thrall and J. G. Pounds, Toxicol. Sci. 2006, 95, 300-312.

7 P. M. Hinderliter, K. R. Minard, G. Orr, W. B. Chrisler, B. D. Thrall, J. G. Pounds and J. G. Teeguarden, Part. Fibre Toxicol. 2010, 7, 36.

8 E. C. Cho, Q. Zhang and Y. Xia, Nat. Nanotech. 2011, 6, 385-391.

9 I. Lynch and K. A. Dawson, Nano Today, 2008, 3, 40-47.

10 E. Mahon, A. Salvati, F. Baldelli Bombelli, I. Lynch and K. A. Dawson, J. Control. Release, 2012, 161, 164-174.

11 T. Cedervall, I. Lynch, S. Lindman, T. Berggard, E. Thulin, H. Nilsson, K. A. Dawson and S. Linse, Proc. Nat. Acad. Sci. U.S.A. 2007, 104, 2050-2055.

12 K. Rausch, A. Reuter, K. Fischer and M. Schmidt, Biomacromolecules, 2010, 11, 2836-2839.

13 W. Jiang, B. Y. S. Kim, J. T. Rutka and W. C. W. Chan, Nat. Nanotech. 2008, 3, 145-150.

14 E. C. Cho, L. Au, Q. Zhang and Y. Xia, Small, 2010, 6, 517-522.

15 D. W. Grainger and D. G. Castner, Adv. Mater., 2008, 20, 867-877.

16 S. E. A. Gratton, P. A. Ropp, P. D. Pohlhaus, J. C. Luft, V. J. Madden, M. E. Napier and J. M. DeSimone, Proc. Nat. Acad. Sci. 2008, 105, 11613-11618.

17 A. Albanese and W. C. Chan, ACS Nano, 2011, 5, 5478-5489.

18 P. Wick, P. Manser, L. K. Limbach, U. Dettlaff-Weglikowska, F. Krumeich, S. Roth, W. J. Stark and A. Bruinink, Toxicol. Lett. 2007, 168, 121-131.

19 P. Meakin, Adv. Colloid Interface Sci. 1988, 28, 249-331.

20 L. K. Limbach, Y. Li, R. N. Grass, T. J. Brunner, M. A. Hintermann, M. Muller, D. Gunther and W. J. Stark, Environ. Sci. Technol. 2005, 39, 9370-9376.

21 G. Sharma, V. Kodali, M. Gaffrey, W. Wang, K. R. Minard, N. J. Karin, J. G. Teeguarden and B. D. Thrall, Nanotoxicology, 2014, 8, 663-675.

22 J. Cohen, G. DeLoid, G. Pyrgiotakis and P. Demokritou, Nanotoxicology, 2013, 7, 417-431.

23 R. Ahmad Khanbeigi, A. Kumar, F. Sadouki, C. Lorenz, B. Forbes, L. A. Dailey and H. Collins, J. Control. Release, 2012, 162, 259-266.

24 R. Pecora, Dynamic Light Scattering, Springer US, Boston, MA, 1985.

25 S. Balog, L. Rodriguez-Lorenzo, C. A. Monnier, B. Michen, M. Obiols-Rabasa, L. Casal-Dujat, B. Rothen-Rutishauser, P. Schurtenberger and A. Petri-Fink, In Preparation.

26 J. Turkevich, P. C. Stevenson and J. Hillier, Discuss. Faraday Soc., 1951, 11, 55.

27 B. J. Marquis, M. A. Maurer-Jones, K. L. Braun and C. L. Haynes, Analyst, 2009, 134, 2293.
28 J. C. Love, L. A. Estroff, J. K. Kriebel, R. G. Nuzzo and G. M. Whitesides, Chem. Rev. 2005, 105, 1103-1170.

29 Z. Sun, W. Ni, Z. Yang, X. Kou, L. Li and J. Wang, Small, 2008, 4, 1287-1292.

30 L. M. Liz-Marzán, Langmuir, 2006, 22, 32-41.

31 P. Sandkuhler, M. Lattuada, H. Wu, J. Sefcik and M. Morbidelli, Adv. Colloid Interface Sci. 2005, 113, 65-83.

32 M. Y. Lin, H. M. Lindsay, D. A. Weitz, R. C. Ball, R. Klein and P. Meakin, Nature, 1989, 339, 360-362.

33 V. Chechik and R. M. Crooks, Langmuir, 1999, 15, 6364-6369.

34 P. Taladriz-Blanco, N. J. Buurma, L. Rodríguez-Lorenzo, J. PérezJuste, L. M. Liz-Marzán and P. Hervés, J. Mater. Chem. 2011, 21, 16880.

35 X. Cai, H.-H. Chen, C.-L. Wang, S.-T. Chen, S.-F. Lai, C.-C. Chien, Y.-Y. Chen, I. M. Kempson, Y. Hwu, C. S. Yang and G. Margaritondo, Anal. Bioanal. Chem. 2011, 401, 809-816.

36 G. Paradossi, F. Cavalieri, E. Chiessi, C. Spagnoli and M. K. Cowman, J. Mater. Sci. Mater. Med. 2003, 14, 687-691.

37 A. Petri-Fink, B. Steitz, A. Finka, J. Salaklang and H. Hofmann, Eur. J. Pharm. Biopharm. 2008, 68, 129-137.

38 V. Hirsch, C. Kinnear, M. Moniatte, B. Rothen, M. J. D. Clift and A. S. Fink, Nanoscale, 2012.

39 S. D. Conner and S. L. Schmid, Nature, 2003, 422, 37-44.

40 Tandon and Rosner, J. Colloid Interface Sci. 1999, 213, 273-286.

41 T. Witten and L. Sander, Phys. Rev. Lett. 1981, 47, 1400-1403.

42 T. Zhu, Z. Jiang and Y. Ma, Appl. Phys. Lett. 2013, 102, 153109.

43 T. dos Santos, J. Varela, I. Lynch, A. Salvati and K. A. Dawson, Small, 2011, 7, 3341-3349.

44 A.-H. Lu, E. L. Salabas and F. Schüth, Angew. Chem. Int. Ed. 2007, 46, 1222-1244.

45 M. Roser, D. Fischer and T. Kissel, Eur. J. Pharm. Biopharm. 1998, 46, 255-263.

46 H. Saïdi, C. Charpentier, H. Bouhlal, D. Schols, J. Balzarini, T. W. Bell, G. Vanham and L. Bélec, Aids Res. Ther. 2010, 7, 16.

47 A. I. Nesvizhskii, A. Keller, E. Kolker and R. Aebersold, Anal. Chem. 2003, 75, 4646-4658. 